\begin{tabular}{|c|c|c|}
\hline Beitr. Ent. & Keltern & ISSN 0005-805X \\
\hline $\mathbf{6 2}(2012) 2$ & S. $287-290$ & 20.12 .2012 \\
\hline
\end{tabular}

\title{
Dr. Reinhard Gaedike zum 70. Geburtstag
}

Mit 1 Figur

\section{ECKHARD K. GROLL}

Am 22. September 2012 vollendete Dr. Reinhard Gaedike das 70. Lebensjahr. An diesem Tag konnte nach jahrelanger Arbeit der „Index Novus Litterarurae Entomologicae“, die vollständig überarbeitete Neuauflage des 1928-1929 von Walther Horn und Sigmund Schenkling herausgegebenen „Index Litteraturae Entomologicae, Serie I“ (Index I) als Online-Datenbank veröffentlicht werden (siehe Seite 459-465 in diesem Heft).

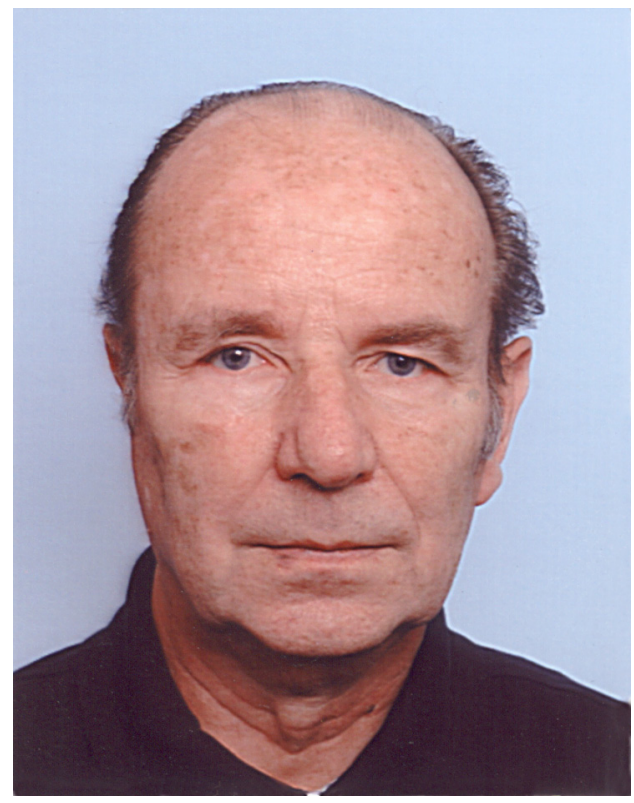

Die Saat zu diesem wichtigen Vorhaben wurde bereits 1965 mit Reinhard Gaedikes Eintritt ins Deutsche Entomologische Institut gelegt. Auf Wunsch Hans Sachtlebens wurde er damals in die Abteilung „Bibliothek, Redaktion der Beiträge und entomologische Bibliographie“ eingegliedert (Petersen, Groll \& Dathe 2008).

Er musste somit seine Arbeitskraft gut einteilen, um neben der wissenschaftlichen Bearbeitung der geliebten Kleinschmetterlinge auch bibliografische und biografische Aufgaben erfüllen zu können. Auf bibliografischem Gebiet publizierte er alsbald die „Bibliographie der Bestimmungstabellen europäischer Insekten“, später „Bibliography of keys to European insects“ (1964 bis 2000). Gemeinsam mit Oldřich Smetana veröffentlichte Reinhard Gaedike 1978 die „Ergänzungen und Berichtigungen zu Walter Horn und Sigmund Schenkling: Index Litteraturae Entomologicae, Serie I, die Weltliteratur über die gesamte Entomologie bis inklusive 1863“. 
Die Versorgung der Entomologen mit historischer und aktueller Literatur war ihm immer sehr wichtig. Ab 1990 mit der Leitung der Bibliothek des DEI betraut, kaufte er mit Mitteln der DFG fehlende Ausgaben von Zeitschriften und Serien und schloss damit Lücken im Bestand der entomologischen Spezialbibliothek des Instituts. Er führte den Zeitschriftentausch auf ein neues Niveau und betrieb mit Antiquariaten im In- und Ausland regen Sondertausch. Forderten Kollegen Literatur mit manchmal kryptischen Zitationen an, recherchierte er erfolgreich. Dabei reiften auch die Ideen des Nachdrucks von Index I, Index II oder gar deren Fortsetzung heran. Mitte der 1990er Jahre gab Reinhard Gaedike schließlich den Anstoß für die Neuauflage des Index I.

Ziel war es, die gesamte entomologische Literatur bis 1863 und die sich darauf beziehenden späteren bibliographischen Arbeiten, gestützt auf eine Datenbank, zu erfassen und den Index I neu herauszugeben. Ab 1997 bearbeiteten Reinhard Gaedike, Eckhard Groll und Andreas Taeger dieses Thema. Eine Datenbank wurde mittels Paradox angelegt und laufend angepasst. Der Autor saß Tür an Tür mit Reinhard Gaedike, sodass viele Ideen zur Datenerfassung und Präsentation schnell mal ausprobiert, verworfen oder implementiert werden konnten.

Ursprünglich sollte der „Index Novus Litteraturae Entomologicae“ noch vor dem verdienten Ruhestand von Reinhard Gaedike als gedrucktes Buch erscheinen. Bis zu diesem Zeitpunkt hatte er Hunderte Originalquellen in den Bibliotheken von London, Berlin, München, Greifswald und Eberswalde recherchiert und sämtliche Zeitschriftentitel anhand der Zeitschriftendatenbank der Staatsbibliothek zu Berlin (ZDB-OPAC) geprüft und korrigiert. Die Endkorrektur der mehr als 1500 Seiten hat er dann nach seinem Ausscheiden aus dem DEI in Bonn vollendet.

Mit der Freigabe der Datenbank muss der Name Reinhard Gaedike nun in einer Reihe mit Walter Horn, Sigmund Schenkling, Hans Sachtleben sowie den Autoren des „Index Litteraturae Entomologicae, Serie II: Die Welt-Literatur über die gesamte Entomologie von 1864 bis 1900“, Walter Derksen und Ursula Göllner (siehe Seite 259-262 in diesem Heft) genannt werden.

Über sein Wirken bei den Kleinschmetterlingen ist in KLAUsNITZER und Nuss (2008) ausfühlich berichtet worden. Diese Arbeiten setzt Reinhard Gaedike kontinuierlich und verstärkt fort. Alljährlich publiziert er Nachträge und Korrekturen zur Faunistik der deutschen Kleinschmetterlinge in der Zeitschrift „Entomologische Nachrichten und Berichte“. Ein Manuskript für den ersten Teilband (270 Arten Tineidae) der Buchreihe „Microlepidoptera of Europe“ entsteht ebenfalls und dürfte im nächsten Jahr in den Druck gelangen. Außerdem arbeitet er am Manuskript der aktualisierten Auflage des Verzeichnisses der Schmetterlinge Deutschlands, da sich seit dem Erscheinen 1999 zahlreiche Änderungen ergeben haben (Nuss 2012).

Wir möchten Reinhard Gaedike alles Gute, vor allem Gesundheit wünschen, das er die sich selbst gesteckten Ziele erreichen kann!

\section{Verzeichnis der Publikationen von Reinhard Gaedike (Fortsetzung nach 2007)}

Gaedike, R. (Hrsg.) 2008: Microlepidoptera Palaearctica, Begr. von H. G. Amsel; F. Gregor; H. Reisser. - Goecke \& Evers, Keltern 13: 558 S., 174 Taf. (Gozmány, L.: Symmocidae).

Gaedike, R.; Kuroko, H. \& Funahashi, K. 2008: A new species of Sinicaepermenia (Lepidoptera, Epermeniidae) from Thailand. - Transactions of the lepidopterological Society of Japan 59 (2): 149-153, 13 figs.

Gaedike, R. 2008: On the generic affiliation of the taxon efflexa XIAO \& Li, 2006 (Tineidae). - Nota lepidopterologica 31 (1): 77. 
Gaedike, R. 2008: Nota lepidopterologica indices to volumes 1 to 30 by taxon and author names, with publication dates. - Nota lepidopterologica 31 (1): 79-162.

GaEdike, R. 2008: Nachträge und Korrekturen zu: Verzeichnis der Schmetterlinge Deutschlands (Microlepidoptera). - Entomologische Nachrichten und Berichte 52 (1): 9-49.

Karisch, T. \& Gaedike, R. 2008: Reinhard Sutter - 70 Jahre. - Entomologische Nachrichten und Berichte 52 (1): 72-73.

GAEDike, R. 2008: New species and records of the Nearctic Epermeniidae (Lepidoptera). - Tijdschrift voor Entomologie 151 (1): 57-64, 24 figs.

Gaedike, R. \& Baldizzone, G. 2008: Records of Lepidoptera Tineidae, Epermeniidae, and Acrolepiidae, from Krk Island (Croatia). - Entomologia Croatica 12 (1): 65-80, 2 figs.

Gaedike, R. 2008: Does Epermenia illigerella (Hübner, 1813) (Lepidoptera: Epermeniidae) occur in the British Isles? - Entomologist's Gazette 59: 240-242, 1 fig.

Gaedike, R. \& Behne, L. 2008: Die Zeitschrift „Le Frelon“ - Datierung und Inhalt. - Entomologische Blätter 103/104: 87-122.

GaEdike, R. 2009: Order Lepidoptera, family Tineidae. - Arthropod fauna of the UAE 2: 433-444, 10 pls, 23 figs.

Gaedike, R. 2009: A new synonym of Trichophaga tapetzella (Linnaeus, 1758) (Lepidoptera: Tineidae). Entomologist's Gazette 60: 85-87.

Gaedike, R. 2009: Some New and Interesting "Microlepidoptera" from the Collection of the Zoologisches Forschungsmuseum Alexander Koenig (ZFMK), Bonn (Lepidoptera: Tineidae, Epermeniidae; Acrolepiidae; Douglasiidae). - Bonner zoologische Beiträge 56 (2007): 101-106, 6 figs.

GaEdike, R. 2009: Nemapogon somchetiella Zagulajev, 1961, a new record for the Italian fauna (Lepidoptera: Tineidae). - Rivista Piemontese di Storia Naturale 30: 53-56, 4 figs.

Gaedike, R. 2009: Nachtrag 2008 zum Verzeichnis der Schmetterlinge Deutschlands (Microlepidoptera). Entomologische Nachrichten und Berichte 53 (2): 75-100, 7 Fig.

Gaedike, R. 2009: New or poorly known Tineidae from Mauretania, Morocco, Algeria, and Tunisia (Lepidoptera). - Beiträge zur Entomologie 59 (2): 489-512, 41 figs.

Kristensen, N. P. \& Gaedike, R. 2010: Extraordinary moths and extraordinary moth researcher: An essay review of G. S. Robinson's Biology, distribution and diversity of tineid moths. - Nota lepidopterologica 33 (1): 3-8, 1 fig.

Gaedike, R. 2010: New an poorly known Palaearctic Microlepidoptera (Tineidae, Acrolepiidae, Douglasiidae, Epermeniidae). - Nota lepidopterologica 33 (1): 9-24, 20 figs.

Gaedike, R. \& Tokár, Z. 2010: Pelecystola fraudulentella (Zeller, 1852) discovered in Slovakia, a third locality record (Tineidae). - Nota lepidopterologica 33 (1): 25-29, 8 figs.

Gaedike, R. 2010: Nachtrag 2009 zum Verzeichnis der Schmetterlinge Deutschlands (Microlepidoptera). Entomologische Nachrichten und Berichte 54 (2): 109-122.

GaEdike, R. 2010: New and poorly known Epermeniidae from the Neotropical, Australasian, Oriental and Palaearctic Regions (Lepidoptera). - Beitrag zur Entomologie 60 (1): 57-70, 30 figs.

Gaedike, R. \& Zerafa, M. 2010: New records of Tineidae from the Maltese islands (Lepidoptera) including description of a new species Eudarcia melitensis sp. n. - Bulletin of the Entomological Society of Malta 3: 9-17, 5 figs.

Gaedike, R.; Gomboc, S. \& Lasan, M. 2010: Zur Kenntnis der Tineidae von Slowenien (Lepidoptera). Acta Entomologica Slovenica 18 (2): 87-126, 25 figs.

Gaedike, R.; Sammut, P. \& Seguna, A. 2011: Proterospastis orientalis Petersen, 1959, a species new to the lepidopterofauna of Malta, Greece and the whole of Europe (Lepidoptera: Tineidae, Tineinae). SHILAP Revista de lepidopterologia 39 (153): 37-38, 1 fig.

Gaedike, R. \& Alipanah, H. 2011: The Tineidae in the Lepidoptera collection of the Iranian Research Institute of Plant Protection (IRIPP) (Lepidoptera: Tineidae). - Shilap Revista de Lepidopterologia 39 (155): 273-284, 7 figs. 
Gaedike, R. 2011: New and poorly known Tineidae from the Western Palaearctic (Lepidoptera). - Beiträge zur Entomologie 61 (2): 357-370, 30 figs.

Gaedike, R. \& Mally, R. 2011: On the taxonomic status of Cephimallota angusticostella (Zeller) and C. crassiflavella Bruand (Tineidae). - Nota lepidopterologica 34 (2): 115-130, 36 figs.

Gaedike, R. 2011: A new species of Digitivalva Gaedike, 1970 from Greece (Acrolepiidae). - Nota lepidopterologica 34 (2): 131-136, 13 figs.

Gaedike, R. 2011: Contributions to the knowledge of Palaearctic Tineidae. - Nota lepidopterologica 34 (2): 137-144, 15 figs.

Gaedike, R. 2011: Nachtrag 2010 zum Verzeichnis der Schmetterlinge Deutschlands (Microlepidoptera). Entomologische Nachrichten und Berichte 55 (4): 199-214.

GaEdike, R. 2012: Beitrag zur Kenntnis der Tineiden von Nepal und benachbarter Gebiete (Lepidoptera: Tineidae). - Beiträge zur Entomologie 62 (1): 165-206, 109 Fig.

Gaedike, R. 2012: Dr. Günther Petersen (25.08.1924 - 24.03.2012) zum Gedenken. - Beiträge zur Entomologie 62 (1): 247-258.

\section{Literatur}

Gaedike, R.; Groll, E. K. \& TAeger, A. 2012: Index Novus Litteraturae Entomologicae : Bibliografie der entomologischen Literatur vom Beginn bis einschließlich 1863 : Online-Datenbank - Version 1.0 Senckenberg Deutsches Entomologisches Institut - URL: http://sdei.senckenberg.de/index (10.11.2012).

Klausnitzer, B. \& Nuss, M. 2008: Dr. Reinhard Gaedike zum 65. Geburtstag. - Entomologische Nachrichten und Berichte, Dresden 52 (1): 75-81, Schr.-Verz., Portr.

Nuss, M. 2012: briefliche Mitteilung.

Petersen, G.; Groll, E. K. \& Dathe, H. H. 2008: Reinhard Gaedike zum 65. Geburtstag. - Beiträge zur Entomologie, Keltern 58 (1): 3-13, Schr.-Verz., Foto.

\section{Anschrift des Verfassers:}

Dr. EckHard K. Groll

Senckenberg Deutsches Entomologisches Institut

Eberswalder Str. 90

15374 Müncheberg

Deuschland 